\title{
Development and Analysis of a Folded Shorted-Patch Antenna With Reduced Size
}

\author{
RongLin Li, Senior Member, IEEE, Gerald DeJean, Student Member, IEEE, Manos M. Tentzeris, Senior Member, IEEE, \\ and Joy Laskar, Senior Member, IEEE
}

\begin{abstract}
The length of a wall-shorted rectangular patch antenna can be reduced from $\sim \lambda_{0} / 4$ to $\sim \lambda_{0} / 8$ by a simple folding operation, which results in a stacked shorted-patch (S-P) structure with a resonant frequency that can be controlled by modifying the distance between the stacked (lower and upper) shorted-patches. A theoretical analysis based on a simple transmission-line model is presented and compared with numerical simulations, showing good agreement if the height of the folded patch is much smaller than the patch length. The physical insight of the variation of the resonant frequency for this reduced-size antenna can be understood by considering the antenna as a shorted patch loaded with a capacitor. An experimental verification is carried out for a $15 \mathrm{~mm} \times 15 \mathrm{~mm} \times 6 \mathrm{~mm}$ folded $S$-P antenna prototype designed for the $2.4 \mathrm{GHz}$ ISM band that can achieve a 10-dB return loss bandwidth of $4 \%$ and results in a nearly omni-directional radiation pattern.
\end{abstract}

Index Terms-Antenna theory, microstrip antennas, small antennas.

\section{INTRODUCTION}

$\mathbf{I}$ N MODERN mobile and wireless communications systems, there is an increasing demand for smaller low-cost antennas that can be easily integrated with packaging structures [1], [2]. It is well-known that planar antennas, e.g., a microstrip patch, have a significant number of advantages over conventional antennas, such as low profile, lightweight and low production cost. Nevertheless, in some mobile/wireless applications such as the AMPS/PCS, GSM/DCS, PDC/PHS, IMT 2000, or WLAN in the $2.4 \mathrm{GHz}$ Industrial Scientific and Medical (ISM) band, their physical size may be too large for handheld terminals.

A number of techniques have been proposed to reduce the physical size of a conventional half-wave $\left(\lambda_{0} / 2, \lambda_{0}\right.$ is the guide wavelength in the substrate) patch antenna [3]-[14]. The most straightforward approach is to use a high dielectric constant substrate [3], however, it leads to poor efficiency and narrow bandwidth. A shorting wall has been used to reduce the overall size of the patch antenna to $\lambda_{0} / 4$ [4], while a shorting pin near the feed can reduce the patch size even further [5], [6].

The planar invert-F antenna (PIFA) is one of the most wellknown and documented small patch antennas [7]. Actually, the

Manuscript received January 14, 2002; revised December 10, 2002. This work was supported in part by the Georgia Electronic Design Center (previously known as Yamacraw Design Center) of the State of Georgia, in part by a National Science Foundation (NSF) CAREER Award under contract NSF \#9964761, and in part by the NSF Packaging Research Center.

The authors are with the School of Electrical and Computer Engineering, Georgia Institute of Technology, Atlanta, GA 30332-250 USA (e-mail rlli@ece.gatech.edu).

Digital Object Identifier 10.1109/TAP.2004.823884
PIFA can be viewed as a shorted-patch (S-P) antenna. Therefore, the antenna length of a PIFA is generally less than $\lambda_{0} / 4$. When the shorting post is located at a corner of a square plate, the length of the PIFA can be reduced to $\lambda_{0} / 8$ [8]. The size of a PIFA can be also reduced to less than $\lambda_{0} / 8$ by capacitively loading it [9].

Recent research efforts on the size reduction of patch antennas have focused on the patch-shape optimization to increase the effective electric length of the patch [10]-[14]. A three-layer [10] or two-layer [11], [12] folded rectangular patch may reduce the resonant frequency by $50 \%$ compared with a conventional patch. By notching a rectangular patch, the antenna length can be reduced to less than $\lambda_{0} / 8$ [13]. A printed antenna with a surface area $75 \%$ smaller than a conventional microstrip patch was obtained by incorporating strategically positioned notches near a shorting pin [14].

In this paper, we propose a simple technique for further reducing the size of a conventional S-P antenna [4]. By folding a quarter-wavelength wall-shorted rectangular patch, the antenna length can be reduced to $\sim \lambda_{0} / 8$. The resonant frequency of the folded S-P antenna can be further lowered through a reduction of the distance between the folded patch parallel surfaces (consisting of a lower patch and an upper patch). First, the geometry of the folded S-P antenna is developed and the antenna performance is demonstrated. Then, a theoretical analysis of the impedance characteristics of the folded S-P is presented with comparison to numerical simulations. Finally, an experimental verification is provided for a folded S-P antenna prototype that was designed at the $2.4 \mathrm{GHz}$ ISM band.

\section{DEVELOPMENT OF THE ANTENNA}

It is well-known that a conventional rectangular patch antenna operating at the fundamental mode $\left(\mathrm{TM}_{010}\right.$ mode $)$ has an electrical length of $\sim \lambda_{0} / 2$, as illustrated in Fig. 1(a). Considering that the electric field is zero for the $\mathrm{TM}_{010}$ mode at the middle of the patch, we can short the patch along its middle line with a metal wall without significantly changing the resonant frequency of the antenna. Thus, we get a S-P antenna with an antenna length of $\sim \lambda_{0} / 4$, as shown in Fig. 1(b). Folding the shorted patch (folded S-P) together with the ground plane [see Fig. 1(c)] maintains the total resonant length of the antenna to be $\sim \lambda_{0} / 4$, while the physical length of the antenna gets reduced by almost $50 \%\left(\sim \lambda_{0} / 8\right)$ through the folding operation. It should be emphasized that it is necessary to fold the ground plane as well while folding the shorted patch. Otherwise the folded antenna would look like an S-antenna developed in 


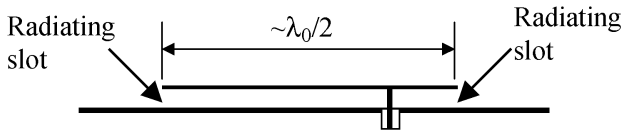

(a)

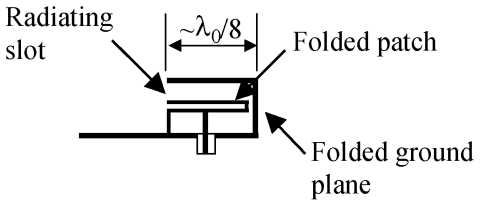

(c)

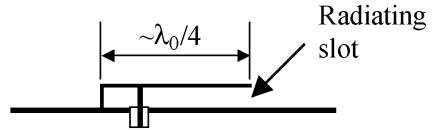

(b)

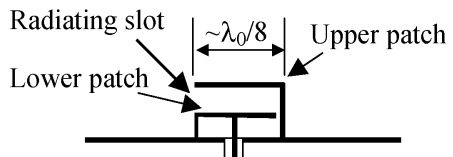

(d)

Fig. 1. Development of a folded S-P antenna. (a) Conventional rectangular patch. (b) Conventional S-P. (c) Folding of a conventional S-P. (d) Folded S-P.

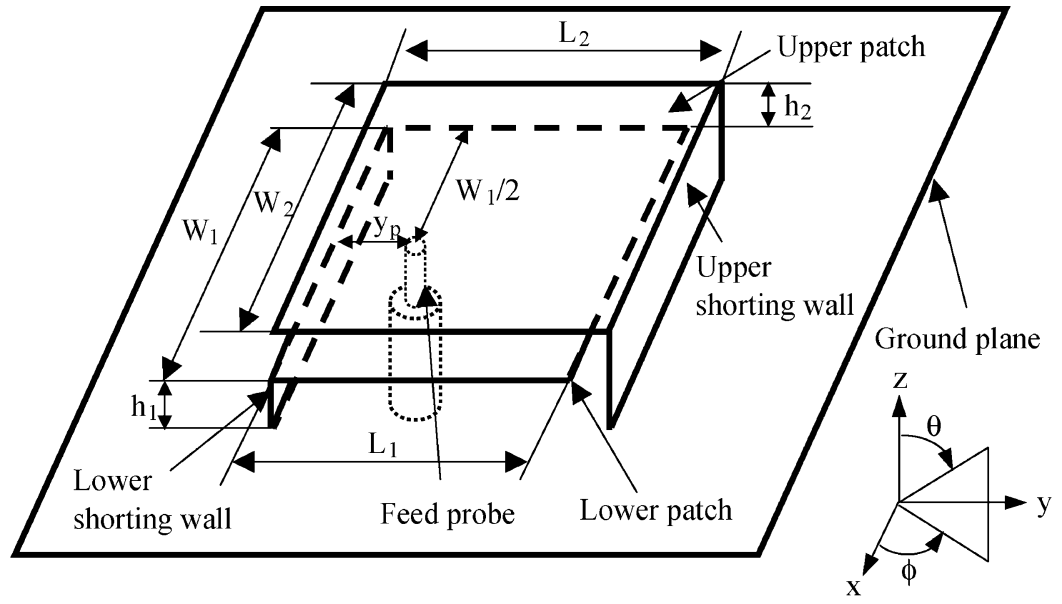

Fig. 2. 3-D geometry of the folded S-P antenna.

[15] for a dual-band operation. Finally, we add a new piece of the ground plane to the right because the original right part of the ground plane now serves as an upper patch of the folded $\mathrm{S}-\mathrm{P}$, and press the folded patch together to form a lower patch of the folded S-P antenna, as shown in Fig. 1(d). The 3-dimensional (3-D) geometry of this folded S-P antenna is illustrated in Fig. 2. The heights, widths and lengths of the lower and upper patches are, respectively, $\mathrm{h}_{1}, \mathrm{~W}_{1}, \mathrm{~L}_{1}$ and $\mathrm{h}_{2}, \mathrm{~W}_{2}, \mathrm{~L}_{2}$. In the analysis below, we assume that the thickness of the patch plates is much smaller than the heights of the patches $\left(h_{1}\right.$ and $\left.h_{2}\right)$. The folded S-P antenna is fed by a probe positioned at the middle of the lower patch in the $x$ direction and $\mathrm{y}_{p}$ away in the $\mathrm{y}$ direction from the edge of the shorting wall of the lower patch.

To demonstrate the size-reduction capability of the folded S-P antenna, we first compare its resonant frequency with that for a conventional S-P antenna, which has the same length and width $(10 \mathrm{~mm} \times 10 \mathrm{~mm})$. The return-loss results $(50 \Omega \mathrm{match})$, simulated by two different simulators, the TLM-based Micro-Stripes $5.6[16]$ and an FDTD code developed in house, are shown in Fig. 3, showing good agreement. It is found that the resonant frequency of the folded S-P (plotted from $2-5 \mathrm{GHz}$ ) is only about $3.6 \mathrm{GHz}$, about $57 \%$ of that $(=6.3 \mathrm{GHz})$ for the conventional S-P antenna. This means that the antenna length of the folded S-P is only $\lambda_{0} / 8, \sim 4$ times smaller than a conventional patch antenna.

It should be mentioned that the resonant frequency of the folded S-P is slightly higher than the expected value, i.e., one half of that for the conventional S-P. This is mainly due to a

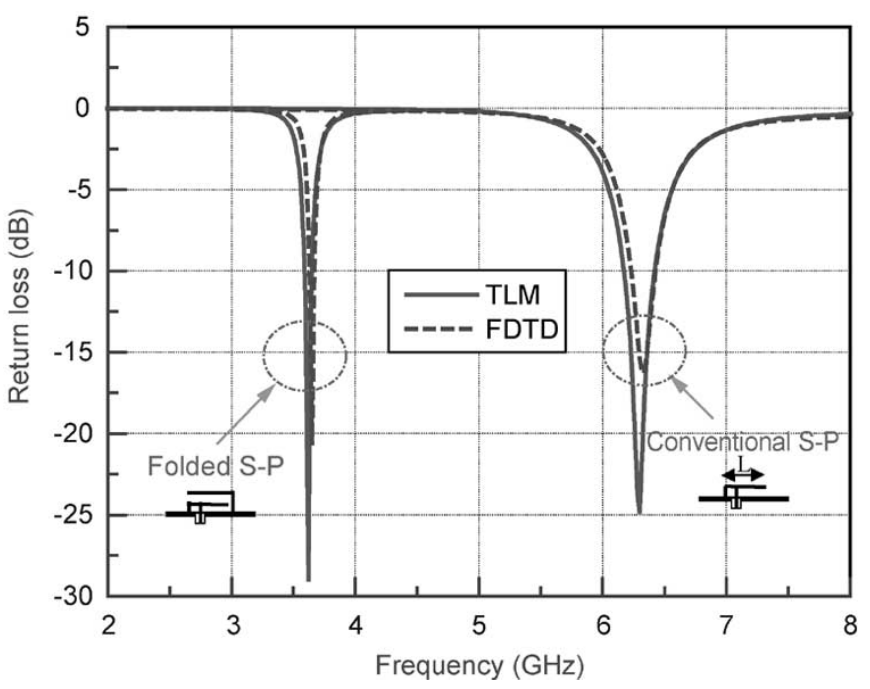

Fig. 3. Return loss for a folded S-P compared with a conventional S-P (parameters for the folded S-P: $\mathrm{L}_{1}=9.5 \mathrm{~mm}, \mathrm{~W}_{1}=10 \mathrm{~mm}, \mathrm{~L}_{2}=\mathrm{W}_{2}=$ $10 \mathrm{~mm}, \mathrm{~h}_{1}=1.5 \mathrm{~mm}, \mathrm{~h}_{2}=1.5 \mathrm{~mm}, \mathrm{y}_{\mathrm{p}}=2.25 \mathrm{~mm}, \mathrm{r}_{\mathrm{p}}=0.25 \mathrm{~mm}$, ground plane $=20 \mathrm{~mm} \times 20 \mathrm{~mm}$; parameters for the conventional S-P: $\mathrm{L}=\mathrm{W}=10 \mathrm{~mm}, \mathrm{~h}=1.5 \mathrm{~mm}, \mathrm{y}_{\mathrm{p}}=2.25 \mathrm{~mm}, \mathrm{r}_{\mathrm{p}}=0.25 \mathrm{~mm}$, ground plane $=20 \mathrm{~mm} \times 20 \mathrm{~mm}$.

shorter length $\left(L_{1}=9.5 \mathrm{~mm}\right)$ of the lower patch of the folded $\mathrm{S}-\mathrm{P}$ than the length $(\mathrm{L}=10 \mathrm{~mm})$ of the conventional S-P because of the need of a small gap between the lower patch and the shorting wall of the upper patch to allow the power flowing from the feeding point to the radiating slot. To support this argu- 


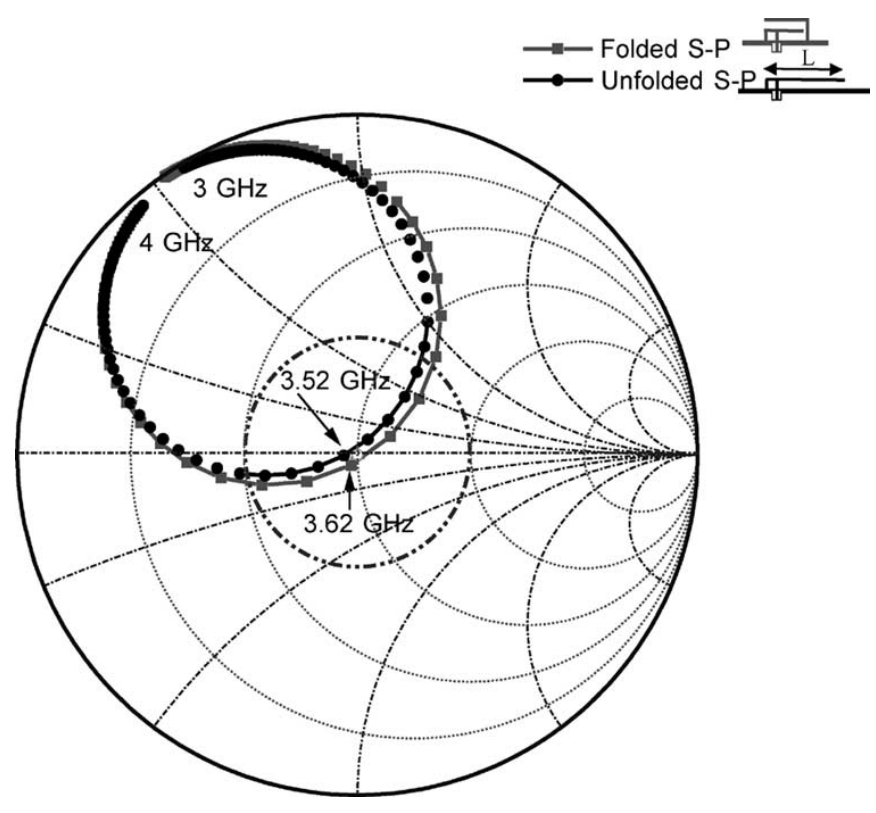

Fig. 4. Comparison of input impedance between a folded S-P and an unfolded S-P (parameters for the folded S-P: $\mathrm{L}_{1}=9.5 \mathrm{~mm}, \mathrm{~W}_{1}=10 \mathrm{~mm}, \mathrm{~L}_{2}=\mathrm{W}_{2}=$ $10 \mathrm{~mm}, \mathrm{~h}_{1}=1.5 \mathrm{~mm}, \mathrm{~h}_{2}=1.5 \mathrm{~mm}, \mathrm{y}_{\mathrm{p}}=2.25 \mathrm{~mm}, \mathrm{r}_{\mathrm{p}}=0.25 \mathrm{~mm}$, ground plane $=20 \mathrm{~mm} \times 20 \mathrm{~mm}$; parameters for the unfolded S-P: L $=19 \mathrm{~mm}$ $\left(=2 \mathrm{~L}_{1}\right), \mathrm{W}=10 \mathrm{~mm}, \mathrm{~h}=1.5 \mathrm{~mm}, \mathrm{y}_{\mathrm{p}}=2.25 \mathrm{~mm}, \mathrm{r}_{\mathrm{p}}=0.25 \mathrm{~mm}$, ground plane $=20 \mathrm{~mm} \times 30 \mathrm{~mm}$ ).

ment, we compare the input impedance of the folded S-P again with a $10 \mathrm{~mm} \times 19 \mathrm{~mm}$ unfolded S-P (whose length $(19 \mathrm{~mm})$ is twice the length $\left(L_{1}=9.5 \mathrm{~mm}\right)$ of the lower patch of the folded $\mathrm{S}-\mathrm{P}$ ) on a Smith chart, as shown in Fig. 4. The agreement of the input impedance between the folded S-P and the unfolded S-P appears to be good, showing a difference of less than $3 \%$ between the resonant frequencies of these two antennas.

To further demonstrate the equivalence of operation between the folded S-P and the unfolded S-P with double physical length, the electric field and surface current distributions at resonant frequencies of the two antennas are compared in Fig. 5. It can be observed that the folded S-P has a field distribution close to that of the unfolded S-P. The electric field of the folded S-P is concentrated in the gap between the lower and upper patches, like the electric-field distribution in the right half part of the unfolded S-P. Also we can observe similar surface current distributions on the folded and unfolded S-P antennas. Note that the lower and upper surfaces of the lower patch of the folded S-P correspond to the lower surface of the unfolded S-P and that the lower surface of the upper patch of the folded S-P corresponds to the right half-part of the ground plane beneath the unfolded S-P.

From the enlarged plot of the electric-field distribution in the folded S-P, we can also observe an electric-field concentration between the edge of the lower patch and the shorting wall of the upper patch due to the effects of a sharp edge of the lower patch and the short distance between the edge and the shorting wall. This electric-field concentration may lead to a reduction of the impedance bandwidth. One way to alleviate it would be an increase in the distance between the edge and the shorting wall, a requirement for a shortened $\mathrm{L}_{1}$. However a decrease in $\mathrm{L}_{1}$ may result in an increase in resonant frequency. For this example, the resonant frequency is $3.6 \mathrm{GHz}$ with a $10-\mathrm{dB}$ return-loss bandwidth of $1.9 \%$ for $L_{1}=9.5 \mathrm{~mm}$, while the resonant frequency increases to $3.8 \mathrm{GHz}$ with an increased bandwidth of $2.1 \%$ if $\mathrm{L}_{1}$ decreases to $8.5 \mathrm{~mm}$, while keeping the length of the upper patch to $\mathrm{L}_{2}=10 \mathrm{~mm}$. Note that the impedance bandwidth of the folded S-P is found to be comparable with that (about 2\%) of the unfolded S-P.

The radiation pattern at the resonant frequency for the folded $\mathrm{S}-\mathrm{P}$ is shown in Fig. 6, where it is compared to a conventional S-P. Note that for comparison, the radiating slots of the folded and conventional S-P antennas are oriented to the same direction, namely, the $-\hat{y}$ direction. As expected, a similar radiation pattern is observed since the folded and conventional S-P antennas share the same radiating slots. Nevertheless, it is noticed that the folded $\mathrm{S}-\mathrm{P}$ has a lower directivity gain (about $3 \mathrm{dBi}$ ) compared to the conventional S-P $(4 \mathrm{dBi})$ due to the increased backside radiation (note that the electric size of the ground plane for the folded S-P is smaller than that for the conventional S-P. The radiation efficiency for the folded S-P is calculated to be $94 \%\left(\sigma=5.8 \times 10^{7} \mathrm{~S} / \mathrm{m}\right)$, slightly lower than its unfolded counterpart (about $96 \%$ ) due to a slightly stronger surface current distribution on the folded S-P and the additional loss on the shorting wall of the upper patch (see Fig. 5). If the resonant frequency of the folded S-P gets smaller by reducing the width of the shorting walls or the distance between the lower and upper patches, the radiation efficiency will reduce significantly. It is found, for example, that for a folded S-P antenna with an antenna length of $\sim \lambda_{0} / 16$ the radiation efficiency is less than $40 \%$.

\section{THEORETICAL ANALYSIS}

The impedance characteristics of the folded S-P antenna can be analyzed by employing a simple transmission-line model [9], [12]. Consider a folded S-P with three different patch-height arrangements: a) case $\mathrm{I}\left(\mathrm{h}_{1}=\mathrm{h}_{2}=1.0 \mathrm{~mm}\right)$; b) case II $\left(\mathrm{h}_{1}=\right.$ $\left.0.5 \mathrm{~mm}, \mathrm{~h}_{2}=1.0 \mathrm{~mm}\right)$; and c) case III $\left(\mathrm{h}_{1}=1.0 \mathrm{~mm}, \mathrm{~h}_{2}=\right.$ $0.5 \mathrm{~mm})$. The equivalent unfolded S-P configurations associated with these three cases are illustrated in Fig. 7(a)-(c). Neglecting the effect of discontinuities because $\left|h_{1}-h_{2}\right|$ is much smaller (at least ten times less) than the length of the folded S-P, the unfolded S-P can be represented by a transmission-line equivalent circuit as shown in Fig. 7(d) [19] with input impedance

$$
Z_{\text {in }}=j X_{f}+Z_{1}
$$

where $X_{f}$ is the feed-probe reactance given by

$$
X_{f}=\frac{\omega \mu_{0} h_{1}}{2 \pi}\left[\ln \left(\frac{2}{\beta r_{p}}\right)-0.57721\right]
$$

with $\beta=2 \pi / \lambda_{0}$ and $r_{p}=$ the feed-probe radius. $\mathrm{Z}_{1}\left(=1 / \mathrm{Y}_{1}\right)$ is obtained from the transmission-line equivalent circuit, that is

$$
\begin{aligned}
& Y_{1}=Y_{01} \frac{1}{j \tan \left(\beta y_{p}\right)}+Y_{01} \frac{Y_{2}+j Y_{01} \tan \left[\beta\left(L_{1}-y_{p}\right)\right]}{Y_{01}+j Y_{2} \tan \left[\beta\left(L_{1}-y_{p}\right)\right]} \\
& Y_{2}=Y_{02} \frac{Y_{s}+j Y_{02} \tan \left(\beta L_{1}\right)}{Y_{02}+j Y_{s} \tan \left(\beta L_{1}\right)}
\end{aligned}
$$

where $Y_{01}$ and $Y_{02}$ are respectively the characteristic admittances of the lower and upper patches, and $Y_{\mathrm{s}}=\mathrm{G}_{\mathrm{s}}+\mathrm{j} \mathrm{B}_{\mathrm{s}}$. Here, $G_{\mathrm{S}}$ is the conductance associated with the power radiated 


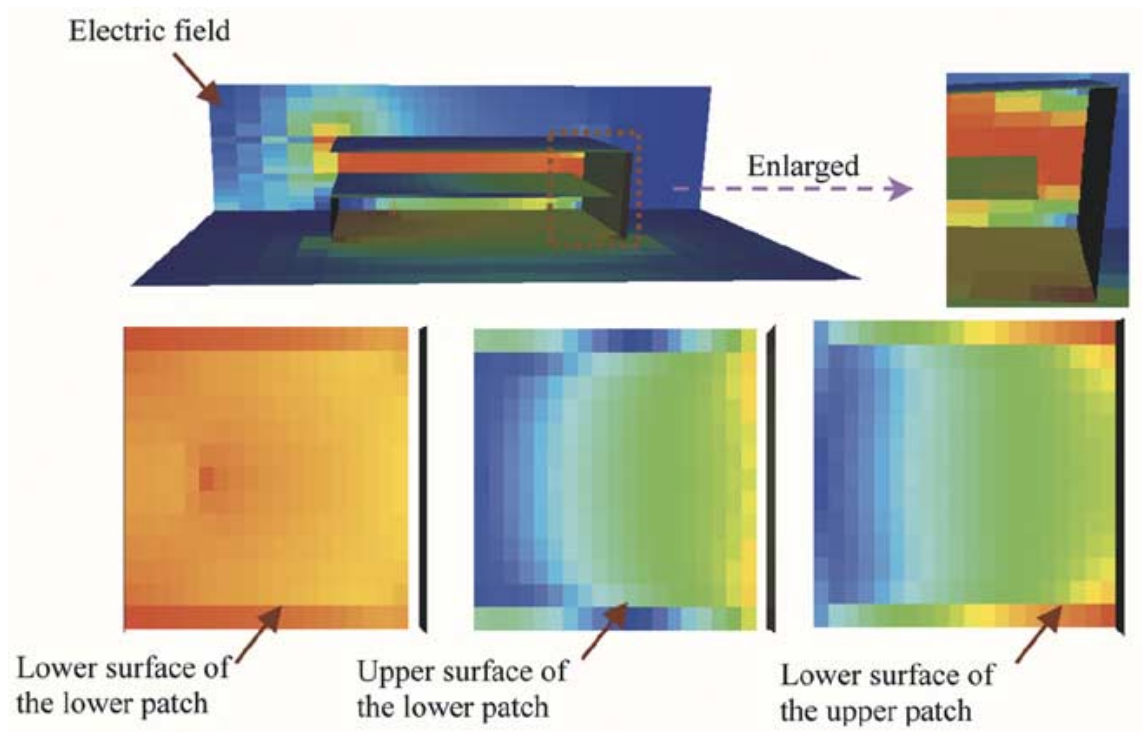

(a) folded S-P
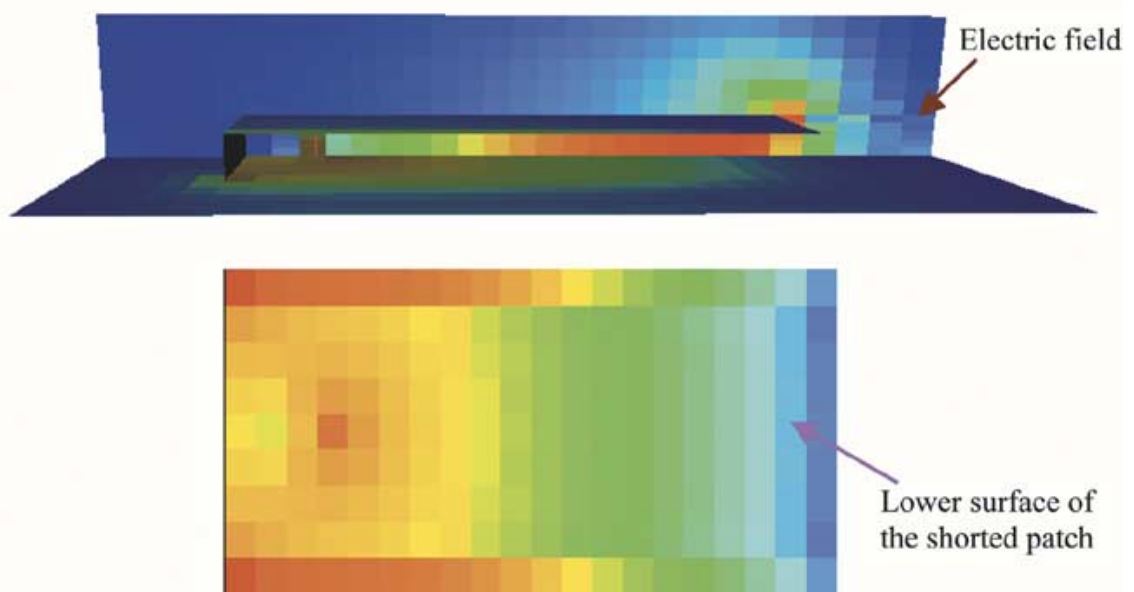

(b) unfolded S-P

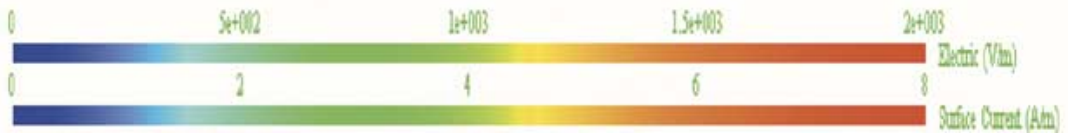

Fig. 5. Electric field and surface current distributions at resonant frequencies. (a) Folded S-P. (b) Unfolded S-P.

from the radiating edge (or the radiating slot), and $B_{\mathrm{S}}$ is the susceptance due to the energy stored in the fringing field near the edge. In our calculations, we used the following equations for $\mathrm{Y}_{0}\left(=\mathrm{Y}_{01}\right.$ for $\mathrm{h}=\mathrm{h}_{1}$ or $\mathrm{Y}_{02}$ for $\left.\mathrm{h}=\mathrm{h}_{2}\right)[20], G_{\mathrm{s}}$, and $B_{\mathrm{s}}$ [19]:

$$
\begin{aligned}
Y_{0} & =\frac{\frac{W}{h}+1.393+0.667 \ln \left(\frac{W}{h}+1.444\right)}{120 \pi} \text { for } \frac{\mathrm{W}}{\mathrm{h}} \geq 1 \\
G_{s} & =\left\{\begin{array}{lc}
\frac{W^{2}}{\left(90 \lambda_{0}^{2}\right)} & \text { for } W \leq 0.35 \lambda_{0} \\
\frac{W}{\left(120 \lambda_{0}\right)}-\frac{1}{\left(60 \lambda_{0}^{2}\right)} & \text { for } 0.35 \lambda_{0} \leq W \leq 2 \lambda_{0} \\
\frac{W}{\left(120 \lambda_{0}\right)} & \left(\mathrm{h}_{2} \leq 0.02 \lambda_{0}\right)
\end{array}\right. \\
B_{s} & =Y_{02} \tan (\beta \Delta I) \\
\Delta l & =\frac{\zeta_{1} \zeta_{3} \zeta_{5}}{\zeta_{4}} h_{2}
\end{aligned}
$$

where $\mathrm{W}$ is the width of the patch and coefficients $\zeta_{1}, \zeta_{3}, \zeta_{4}, \zeta_{5}$ can be found in Appendix B of [19].

The theoretical results for the input impedance have been obtained using the above analytical expressions and compared in Fig. 8 with numerical simulation (using Micro-Stripes 5.6) for the above three cases of the folded S-P, demonstrating a good agreement. The difference between the theoretical and simulated resonant frequencies is less than $3 \%$. Also, it can be observed that the resonant frequency decreases as $h_{2} / h_{1}$ decreases. This can be explained qualitatively as follows:

For simplicity, we neglect the effects of $\mathrm{Y}_{\mathrm{s}}$ (typically $\mathrm{Y}_{\mathrm{s}} \ll$ $\mathrm{Y}_{0}$ [21]) and $X_{f}$ (note that we are now only interested in the resonance of the patch alone). As a result the unfolded $\mathrm{S}$-P becomes a shorted transmission line loaded with an open transmission line. Assuming that the resonant frequency is almost 

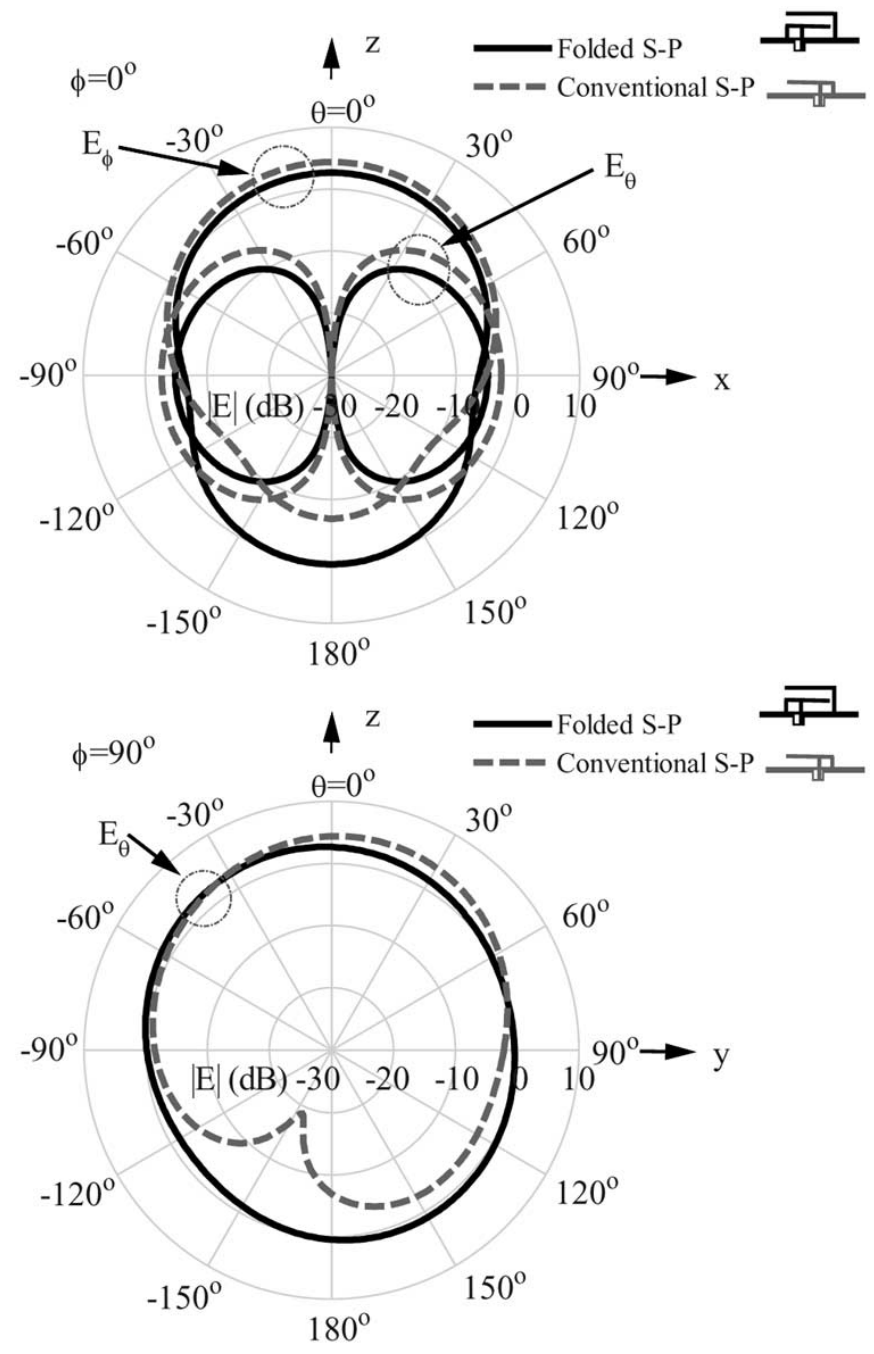

Fig. 6. Calculated radiation pattern of the folded S-P at $3.6 \mathrm{GHz}$ compared with a conventional S-P at $6.3 \mathrm{GHz}$.

independent on the feeding position, we can choose $y_{p}=L_{1}$. Thus, $\mathrm{Y}_{1}$ becomes

$$
Y_{1}=Y_{01} \frac{1}{j \tan \left(\beta L_{1}\right)}+j Y_{02} \tan \left(\beta L_{1}\right)
$$

At resonance, $\mathrm{Y}_{1}=0$ leads to

$$
\frac{Y_{01}}{\tan \left(\beta L_{1}\right)}=Y_{02} \tan \left(\beta L_{1}\right) \text { or } \tan \left(\beta L_{1}\right)=\sqrt{\frac{Y_{01}}{Y_{02}}} \text {. }
$$

From (5), it is observed that $Y_{0}$ is inversely proportional to $h$; therefore, we can find from (10) that the resonant frequency varies proportionally with $h_{2} / h_{1}$. A graphical solution of (10) for resonant frequency is depicted in Fig. 9, where the intersection of the curves $Y_{01} / \tan \left(\beta L_{1}\right)$ and $Y_{02} \tan \left(\beta L_{1}\right)$ implies a resonant point. Observe that if $\mathrm{Y}_{01}=\mathrm{Y}_{02}$, then $\beta \mathrm{L}_{1}=\pi / 4$, which corresponds to an antenna length of $\mathrm{L}_{1}=\lambda_{0} / 8$. Also observe that an increase in $\mathrm{Y}_{02}$ leads to a decrease in $\beta \mathrm{L}_{1}$ if $\mathrm{Y}_{01}$ keeps unchanged.

Considering the upper patch as a capacitive load leads to a clear picture of the physical insight for the above analysis. Replacing the upper patch with a capacitor $\mathrm{C}$ which is connected

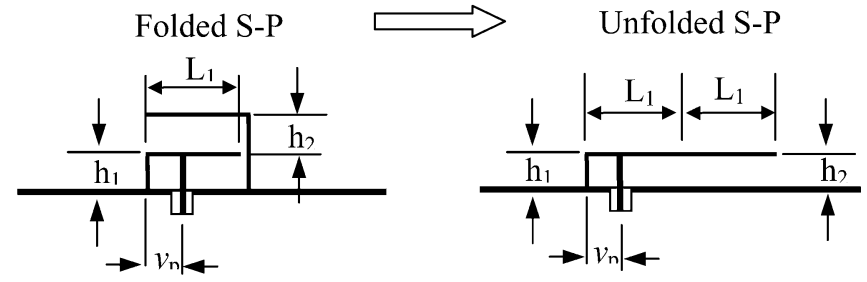

(a)

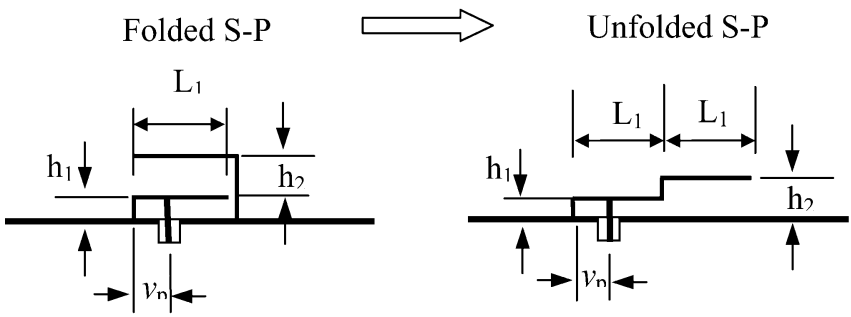

(b)

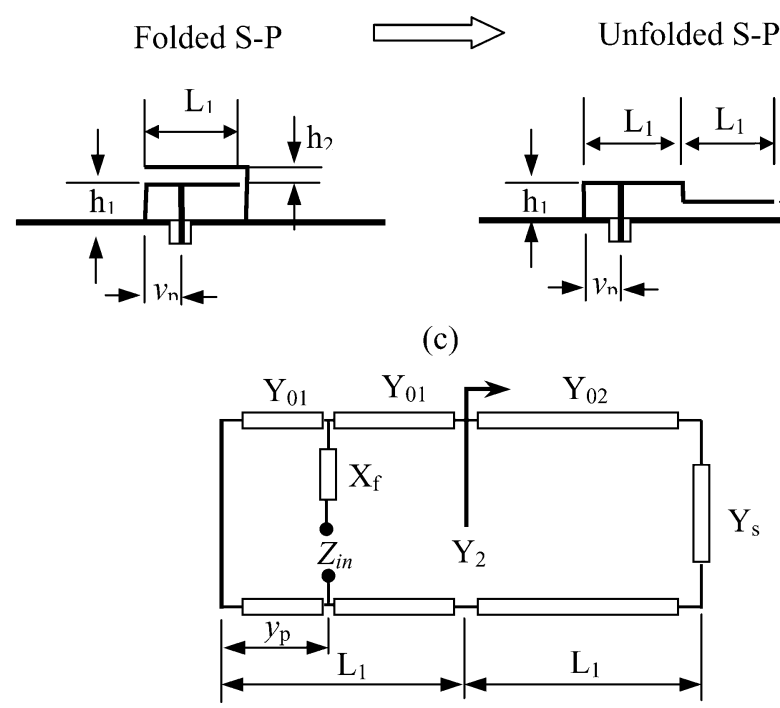

(d)

Fig. 7. Folded S-P and its equivalent transmission-line model. (a) Case $\mathrm{I}\left(\mathrm{h}_{1}=\right.$ $\mathrm{h}_{2}=1.0 \mathrm{~mm}$ ). (b) Case II $\left(\mathrm{h}_{1}=0.5 \mathrm{~mm}, \mathrm{~h}_{2}=1.0 \mathrm{~mm}\right)$. (c) Case III $\left(\mathrm{h}_{1}=1\right.$ $\mathrm{mm}, \mathrm{h}_{2}=0.5 \mathrm{~mm}$ ). (d) Simple transmission-line model.

between the radiating edge of the lower patch and the ground plane, (9) becomes

$$
\frac{Y_{01}}{\tan \left(\beta L_{1}\right)}=\omega C
$$

A graphical solution of (11) is also plotted in Fig. 9. Obviously, the resonant frequency decreases as the capacitance increases. The resonant length of a capacitively loaded S-P will reduce to $\mathrm{L}_{1}=\lambda_{0} / 8$ if the loaded capacitance is $C=Y_{01} / \omega_{0}$, where $\omega_{0}=3 \pi /\left(4 L_{1}\right) \times 10^{8} \mathrm{rad}-\mathrm{s}^{-1}$ obtained from $\beta \mathrm{L}_{1}=\pi / 4$. Actually a decrease in $h_{2}$ is equivalent to an increase in the coupling capacitance between the upper and lower patches, thus eventually leading to a decrease in the resonant frequency. In fact, some of small antenna structures ([9]-[12]) can be considered as a capacitively loaded patch.

It has to be noted that the above simple transmission-line model works well only if the total height of the folded patch is much smaller (at least five times less) than the patch length 


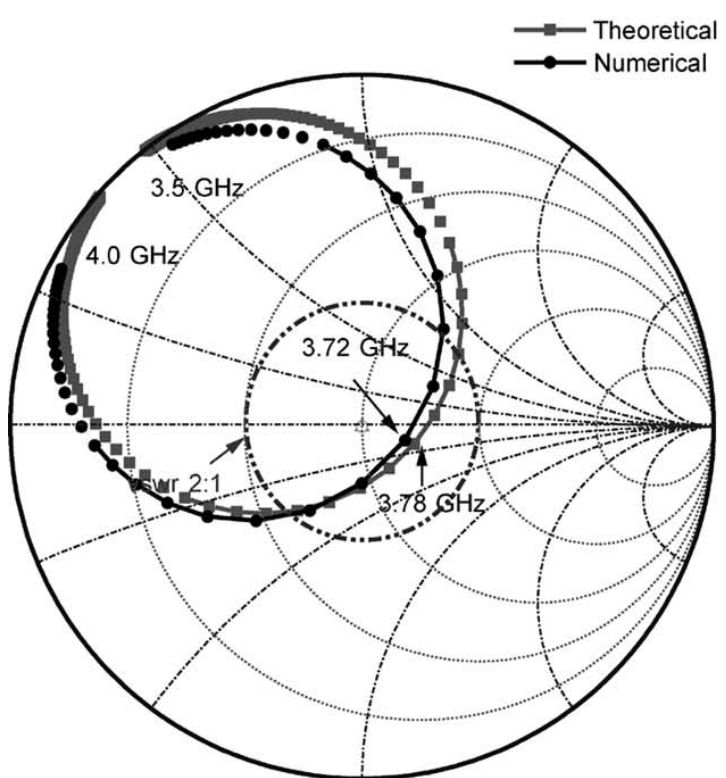

(a)

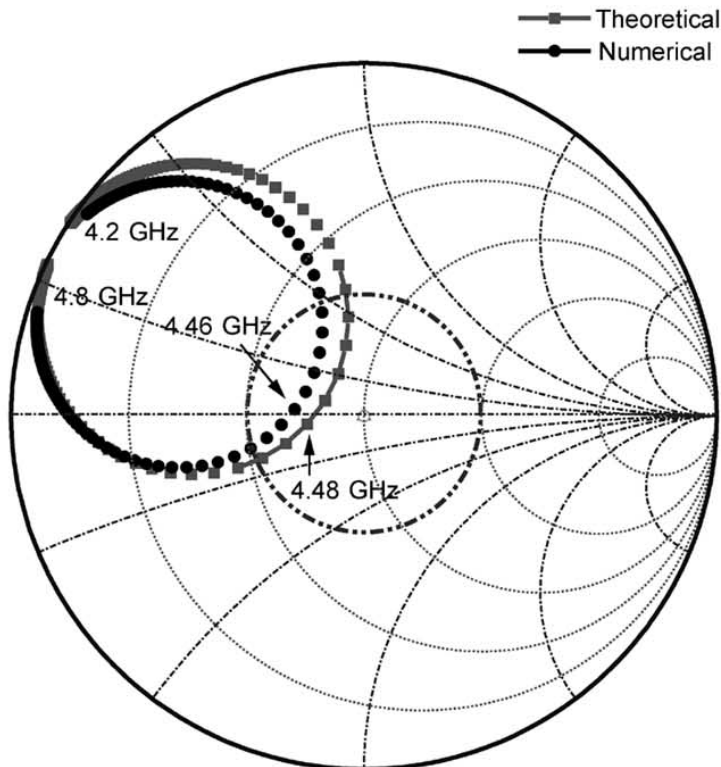

(b)

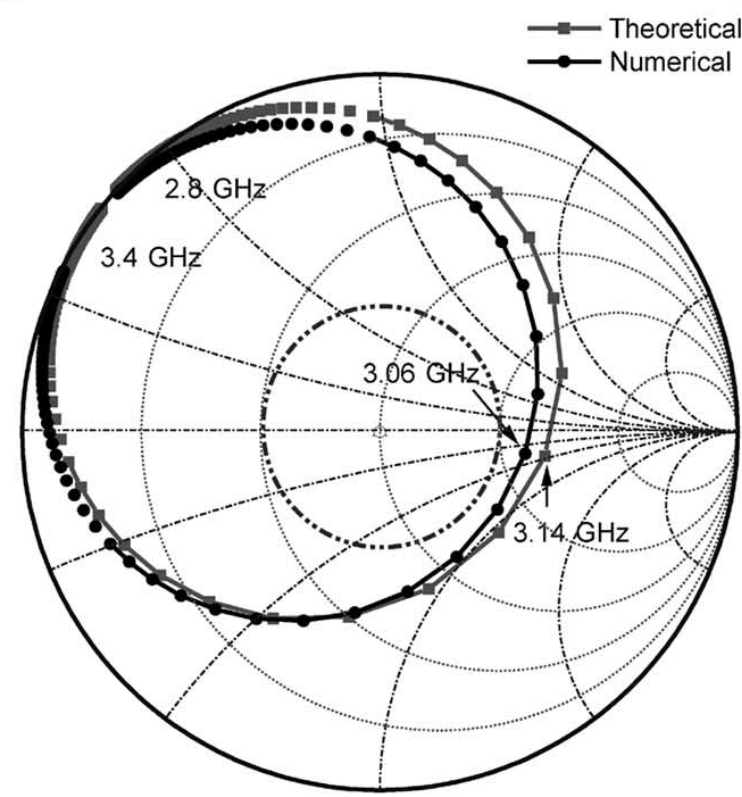

(c)

Fig. 8. Theoretical (transmission-line model) and numerical results for input impedance of a folded S-P with different height arrangements (parameters: $\mathrm{L}_{1}=$ $9.5 \mathrm{~mm}, \mathrm{~W}_{1}=10 \mathrm{~mm}, \mathrm{~L}_{2}=\mathrm{W}_{2}=10 \mathrm{~mm}, \mathrm{y}_{\mathrm{p}}=1.5 \mathrm{~mm}, \mathrm{r}_{\mathrm{p}}=0.25 \mathrm{~mm}$, ground plane $=20 \mathrm{~mm} \times 20 \mathrm{~mm}$ ). (a) Case I $\left(\mathrm{h}_{1}=\mathrm{h}_{2}=1.0 \mathrm{~mm}\right)$. (b) Case II $\left(\mathrm{h}_{1}=0.5 \mathrm{~mm}, \mathrm{~h}_{2}=1.0 \mathrm{~mm}\right)$. (c) Case III $\left(\mathrm{h}_{1}=1.0 \mathrm{~mm}, \mathrm{~h}_{2}=0.5 \mathrm{~mm}\right)$.

and if the discontinuity $\left(\left|\mathrm{h}_{1}-\mathrm{h}_{2}\right|\right)$ is much shorter (at least 10 times less) than the total length of the folded patch antenna.

\section{EXPERIMENTAL VERIFICATION}

A folded S-P antenna prototype has been designed for the 2.4 GHz ISM band (2.4-2.483 GHz). We chose the dimensions of the folded S-P to be $15 \mathrm{~mm} \times 15 \mathrm{~mm}\left(\approx \lambda_{0} / 8 \times \lambda_{0} / 8\right)$. Note that the dimensions of the patch in [22] are $38.6 \mathrm{~mm} \times 37 \mathrm{~mm}$ even though it was built on a substrate with a dielectric constant of 2.23. In order to achieve the bandwidth (near 4\%) required by the ISM band, the total thickness of the antenna was selected to be $6 \mathrm{~mm}$. By adjusting the height $\left(\mathrm{h}_{1}\right)$ of the lower patch to $2.85 \mathrm{~mm}$, we can tune the resonant frequency near $2.44 \mathrm{GHz}$.
This folded S-P antenna was fabricated with of a brass sheet with a thickness of $0.254 \mathrm{~mm}$. The simulated and measured results for the return loss are plotted in Fig. 10 with an inset picture of the antenna prototype. As seen, good agreement is obtained and both of the simulated and measured 10-dB return-loss bandwidths cover the ISM band. The simulated and measured radiation patterns in the $x z$-and $y z$-planes at $2.44 \mathrm{GHz}$ are compared in Fig. 11. Good agreement is again noted. A slight discrepancy is mainly due to the SMA connector used in the measurement, which has a comparable size with the antenna. There is a nearly omni-directional pattern for the copolarized component, which is desirable for mobile/wireless applications. The radiation efficiency of the folded S-P is measured to be approximately $90 \%$, 


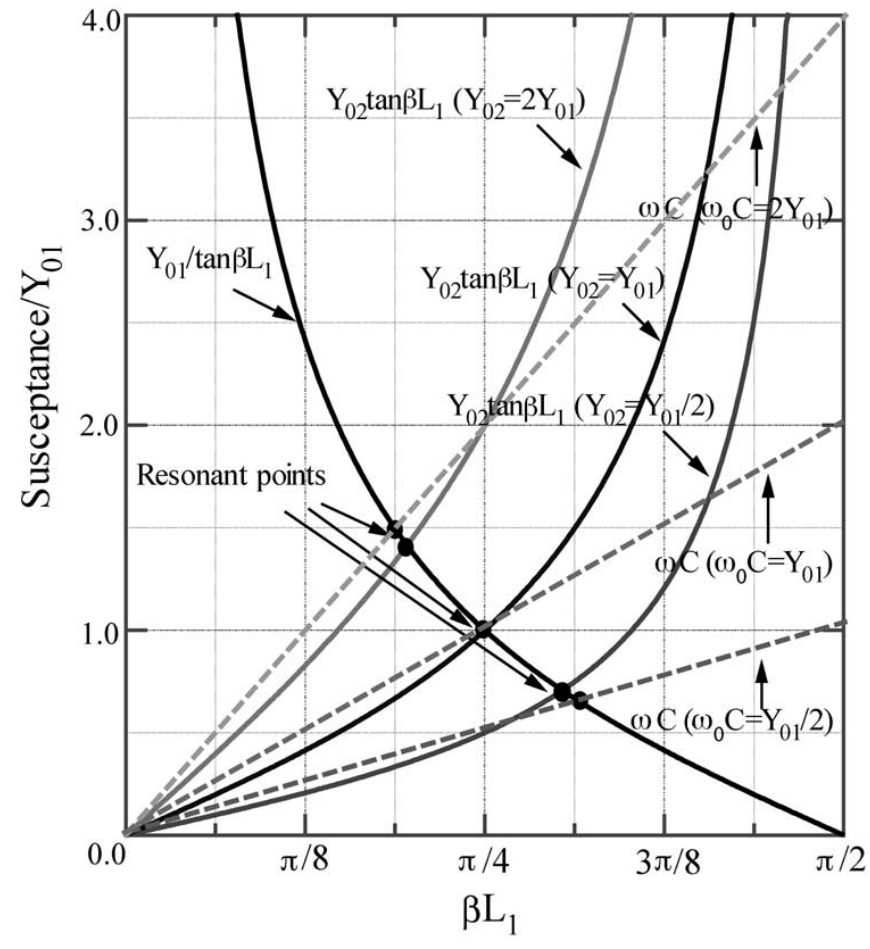

Fig. 9. Graphical solution of (10) and (11) for the calculation of the resonant frequencies of a capacitively loaded S-P $\left(\omega_{0}=3 \pi /\left(4 L_{1}\right) \times 10^{8} \mathrm{rad}_{-} \mathrm{s}^{-1}\right)$.

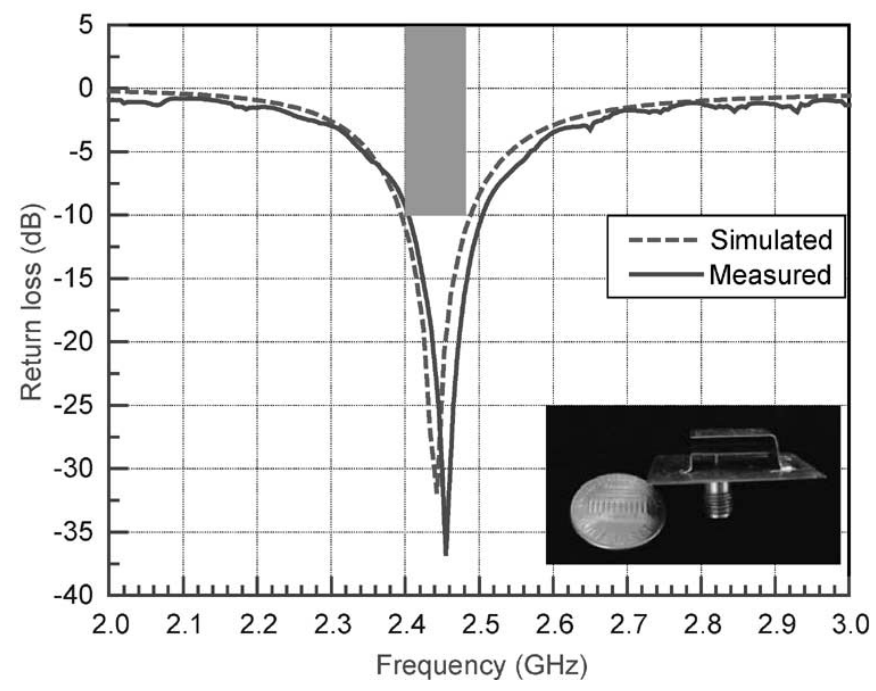

Fig. 10. Simulated and measured results for the return loss of a folded S-P prototype for the $2.4 \mathrm{GHz}$ ISM band (parameters: $\mathrm{L}_{1}=14 \mathrm{~mm}, \mathrm{~L}_{2}=15 \mathrm{~mm}$, $\mathrm{W}_{1}=\mathrm{W}_{2}=15 \mathrm{~mm}, \mathrm{~h}_{1}=2.85 \mathrm{~mm}, \mathrm{~h}_{2}=3.15 \mathrm{~mm}, \mathrm{~d}_{1}=\mathrm{d}_{2}=15 \mathrm{~mm}$, $\mathrm{y}_{\mathrm{p}}=5 \mathrm{~mm}, \mathrm{r}_{\mathrm{p}}=0.325 \mathrm{~mm}$, ground plane $\left.=30 \mathrm{~mm} \times 30 \mathrm{~mm}\right)$.

slightly lower than the simulated result (95\%), mainly due to both calibration and simulation errors.

\section{CONCLUSION}

The length of a conventional quarter-wavelength S-P antenna can be reduced by a factor of almost 2 via folding the shorted patch together with the ground plane. Moving the lower and upper patches closer may further lower the resonant frequency of the folded S-P antenna. A theoretical analysis reveals the physical insight of the resonance characteristics of this antenna.
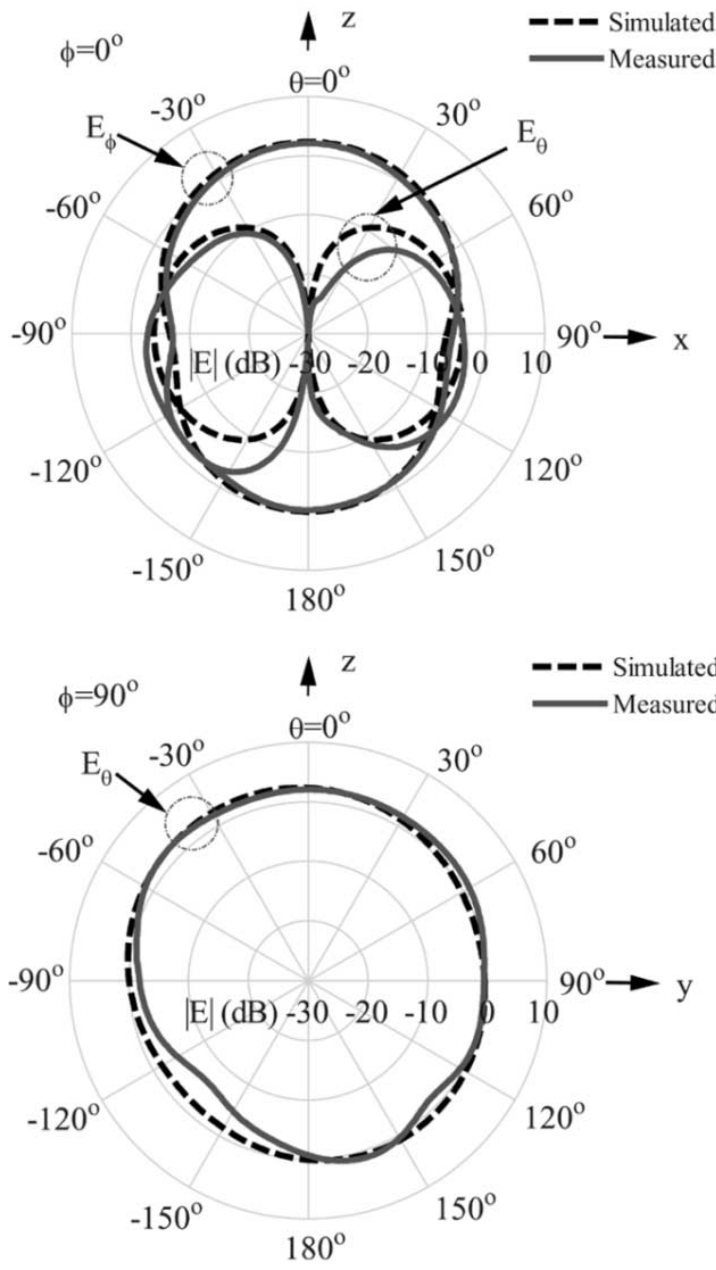

Fig. 11. Comparison of simulated and measured radiation patterns at $2.44 \mathrm{GHz}$ for the folded S-P prototype in Fig. 10.

The upper patch can be considered as a capacitive load, thus resulting in a decrease in the resonant frequency. A prototype of a folded S-P antenna has been designed and fabricated for the 2.4 $\mathrm{GHz}$ ISM band, achieving an impedance bandwidth of $4 \%$ and a nearly omni-directional radiation pattern, which may be suitable for numerous mobile communications/WLAN systems.

\section{ACKNOWLEDGMENT}

The authors would like to acknowledge a number of valuable suggestions and comments from two reviewers.

\section{REFERENCES}

[1] K. Fujimoto, A. Henderson, K. Hirasawa, and J. R. James, Small Antennas. London, U.K.: Research Studies Press, 1987.

[2] A. K. Skrivervik, J.-F. Zurcher, O. Staub, and J. R. Mosig, "PCS antenna design: the challenge of miniaturization," IEEE Antennas Propagat. Mag., vol. 43, pp. 12-27, Aug. 2001.

[3] T. K. Lo, C.-O. Ho, Y. Hwang, E. K. W. Lam, and B. Lee, "Miniature aperture-coupled microstrip antenna of very high permittivity," Electron. Lett., vol. 33, pp. 9-10, Jan. 1997.

[4] S. Pinhas and S. Shtrikman, "Comparison between computed and measured bandwidth of quarter-wave microstrip radiators," IEEE Trans. Antennas Propagat., vol. 36, pp. 1615-1616, Nov. 1988.

[5] R. B. Waterhouse, S. D. Targonski, and D. M. Kokotoff, "Design and performance of small printed antennas," IEEE Trans. Antennas Propagat., vol. 46, pp. 1629-1633, Nov. 1998.

[6] Y. J. Wang, C. K. Lee, W. J. Koh, and Y. B. Gan, "Design of small and broad-band internal antennas for IMT-2000 mobile handsets," IEEE Trans. Microwave Theory Tech., vol. 49, pp. 1398-1403, Aug. 2001. 
[7] T. Taga, "Analysis of planar inverted-F antennas and antenna design for portable radio equipment," in Analysis, Design and Measurement of Small and Low-Profile Antennas, K. Hirasawa and M. Haneishi, Eds. Norwood, MA: Artech House, 1992, pp. 161-180.

[8] K. Ogawa and T. Uwano, "A diversity antenna for very small $800-\mathrm{MHz}$ band portable telephone," IEEE Trans. Antennas Propagat., vol. 42, pp. 1342-1345, Sept. 1994.

[9] C. R. Rowell and R. D. Murch, "A capacitively loaded PIFA for compact mobile telephone handsets," IEEE Trans. Antennas Propagat., vol. 45, pp. 837-842, May 1997.

[10] K. M. Luk, R. Chair, and K. F. Lee, "Small rectangular patch antenna," Electron. Lett., vol. 34, pp. 2366-2367, Dec. 1998.

[11] R. Chair, K. M. Luk, and K. F. Lee, "Small dual patch antenna," Electron. Lett., vol. 35, pp. 762-763, May 1999.

[12] - "Measurement and analysis of miniature multilayer patch antenna," IEEE Trans. Antennas Propagat., vol. 50, pp. 244-250, Feb. 2002.

[13] K.-L. Wong and K.-P. Yang, "Modified planar inverted F antenna," Electrons. Lett., vol. 34, pp. 7-8, Jan. 1998.

[14] H. K. Kan and R. B. Waterhouse, "Size reduction technique for shorted patches," Electron. Lett., vol. 35, pp. 948-949, June 1999.

[15] L. Zaid, G. Kossiavas, J.-Y. Dauvignac, J. Cazajous, and A. Papiernik, "Dual-Frequency and broad-band antennas with stacked quarter wavelength elements," IEEE Trans. Antennas Propagat., vol. 47, pp. 654-660, Apr. 1999.

[16] Electromagnetics Division of Flomerics, Micro-Stripes Version 5.6.0, 2000.

[17] W. F. Richard, Y. T. Lo, and D. D. Harrison, "An improved theory for microstrip antennas and applications," IEEE Trans. Antennas Propagat., vol. 29, pp. 38-46, Jan. 1981.

[18] P. Perlmutter, S. Shritkman, and D. Treves, "Electric surface current model for the analysis of microstrip antennas with application to rectangular elements," IEEE Trans. Antennas Propagat., vol. 33, pp. 301-311, 1985.

[19] R. Garg, P. Bhartia, I. Bahl, and A. Ittipiboon, Microstrip Antenna Design Handbook. Norwood, MA: Artech House, 2001.

[20] D. M. Pozar, Microwave Engineering. New York: Addison-Wesley, 1990, p. 185

[21] C. A. Balanis, Antenna Theory: Analysis and Design. New York: Wiley, 1997, p. 734.

[22] G. Vermeeren, H. Rogier, F. Olyslager, and D. De Zutter, "Simple low-cost planar antenna for indoor communication under the Bluetooth protocol," Electron. Lett., vol. 37, pp. 1153-1154, Sept. 2001.

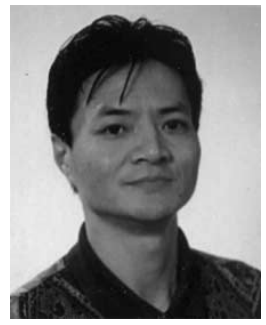

RongLin Li (M'02-SM'03) received the B.S. degree in electrical engineering from Xi' an Jiaotong University, X'an, China, in 1983 and the M.S. and Ph.D. degrees in electrical engineering from Chongqing University, Chongqing, China, in 1990 and 1994, respectively.

From 1983 to 1987, he worked as an Electrical Engineer at Yunnan Electric Power Research Institute, Yunnan, China. From 1994 to 1996, he was a Postdoctoral Research Fellow at Zhejiang University, Zhejiang, China, where in 1998, he became a Professor. In 1997, he was a Research Fellow at Hosei University, Hosei, Japan. In 2000, he was a Research Fellow at Queen's University of Belfast, U.K. Currently, he is a Research Scientist at the Georgia Institute of Technology, Atlanta. His latest research interests include computational electromagnetics and modeling of antennas and microwave devices for wireless communications.

Dr. Li received a Hosei International Fund Research Fellowship in 1997.

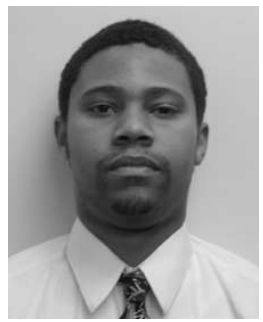

Gerald DeJean ( $\left.\mathrm{S}^{\prime} 03\right)$ was born in Los Angeles, CA. He received the B.S. degree in electrical engineering from Michigan State University, East Lansing, in 2000 and is currently working toward the Ph.D. degree in electromagnetics at the Georgia Institute of Technology, Atlanta.

His main research interests are antenna design, microwave design, and numerical analysis.

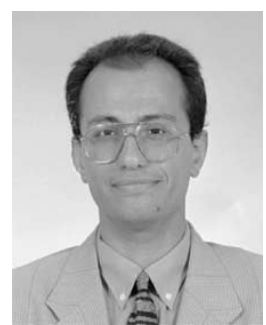

Manos M. Tentzeris (S'89-M'98-SM'03) received the Diploma Degree from the National Technical University of Athens, Greece, and the M.S. and $\mathrm{Ph} . \mathrm{D}$. degrees in electrical engineering and computer science from the University of Michigan, Ann Arbor.

In summer 2002, he was a Visiting Professor with the Technical University of Munich, Germany. Currently, he is an Associate Professor with the School of Electrical and Computer Engineering, Georgia Institute of Technology, Atlanta. He is the Georgia Tech NSF-Packaging Research Center Associate Director for RF Research, the RF Alliance Leader at the Georgia Institute of Technology National Science Foundation (NSF)-Packaging Research Center and the Leader of the Novel Integration Techniques Subthrust of the Broadband Hardware Access Thrust of the Yamacraw Research Initiative, State of Georgia. He has published four book chapters and more than 140 papers in refereed journals and conference proceedings. He has helped develop academic programs in highly integrated packaging for RF and wireless applications, microwave MEMs, SOP-integrated antennas, and adaptive numerical electromagnetics (FDTD, multiresolution algorithms).

Dr. Tentzeris is a Member of the International Scientific Radio Union (URSI)-Commission D and a Member of the Technical Chamber of Greece. He was the recipient of the 1997 Best Paper Award, International Hybrid Microelectronics and Packaging Society, the 2000 NSF CAREER Award, the 2001 ACES Conference Best Paper Award, the 2002 Georgia Tech-ECE Outstanding Junior Faculty Award, the 2002 International Conference on Microwave and Millimeter-Wave Technology Best Paper Award (Beijing, China), the 2003 IEEE CPMT "Outstanding Young Engineer Award," and the 2004 IBA International Educator of the Year. He was also the 1999 Technical Program Co-Chair of the 54th ARFTG Conference, Atlanta, GA and he is the Vice-Chair of the RF Technical Committee (TC16) of the IEEE CPMT Society.

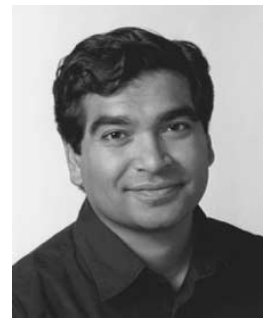

Joy Laskar (S'84-M'85-SM'02) received the B.S. degree in computer engineering with math/physics minors (highest honors) from Clemson University, Clemson, SC, in 1985 and the M.S. and Ph.D. degrees in electrical engineering from the University of Illinois at Urbana-Champaign, in 1989 and 1991, respectively.

Previously, he held faculty positions at the University of Illinois and the University of Hawaii. In 1995, he joined the Georgia Institute of Technology, Atlanta, where since July 2001, he has been named the Joseph M. Pettit Professor of Electronics in the School of Electrical and Computer Engineering and is the Chair for the Electronic Design and Applications Technical Interest Group, the Director of Research for the State of Georgia's Yamacraw Design Center, and the System Research Leader for the National Science Foundation (NSF) Packaging Research Center. At the Georgia Institute of Technology, he heads a research group of 25 members with a focus on integration of high frequency electronics with optoelectronics and integration of mixed technologies for next-generation wireless and optoelectronic systems. In 1998, he cofounded RF Solutions, an advanced WLAN company, which is a recognized leader in the development of 802.11a/b/g RFICs. In 2001, he cofounded Quellan, a next-generation interconnect company that is developing collaborative signal processing solutions for enterprise applications. In addition, he is a Technical Advisor to numerous industry and investor groups. He has authored or coauthored more than 200 papers, several book chapters (including three textbooks in development), numerous invited talks, and has 10 patents pending. His research has produced numerous patents and transfer of technology to industry. His research has focused on high frequency IC design and their integration.

Dr. Laskar is a recipient of the 1995 Army Research Office's Young Investigator Award, a 1996 recipient of the NSF CAREER Award, the 1997 NSF Packaging Research Center Faculty of the Year, the 1998 NSF Packaging Research Center Educator of the Year, the 1999 corecipient of the IEEE Rappaport Award (Best IEEE Electron Devices Society Journal Paper), the Faculty Advisor for the 2000 IEEE MTT IMS Best Student Paper award, 2001 Georgia Tech Faculty Graduate Student Mentor of the year, recipient of a 2002 IBM Faculty Award, the 2003 Clemson University College of Engineering Outstanding Young Alumni Award, and the 2003 recipient of the Outstanding Young Engineer of the Microwave Theory and Techniques Society. 